\title{
Reversal of Oral Anticoagulants for Intracerebral Hemorrhage Patients: Best Strategies
}

\author{
Lanting Fuh, PharmD ${ }^{1}$ Jonathan H. Sin, PharmD ${ }^{1}$ Joshua N. Goldstein, MD, PhD ${ }^{2}$ \\ Bryan D. Hayes, PharmD ${ }^{1,2}$ \\ ${ }^{1}$ Department of Pharmacy, Massachusetts General Hospital, Boston, \\ Massachusetts \\ ${ }^{2}$ Department of Emergency Medicine, Harvard Medical School, \\ Boston, Massachusetts \\ Address for correspondence Bryan D. Hayes, PharmD, Department of \\ Pharmacy, Massachusetts General Hospital, 55 Fruit Street, Boston, \\ MA 02114 (e-mail: bryanhayes13@gmail.com).
}

Semin Respir Crit Care Med 2017;38:726-736.

\begin{abstract}
Keywords

- cerebral hemorrhage

- anticoagulants

- warfarin

- dabigatran

- rivaroxaban

In patients with acute intracerebral hemorrhage (ICH), one of the major concerns is ongoing bleeding or $\mathrm{ICH}$ expansion. Anticoagulated patients are at higher risk of ongoing expansion and worse outcome. It may be that rapid anticoagulation reversal can reduce the risk of expansion and improve clinical outcome. For those taking coumarins, the best available evidence suggests that intravenous vitamin $\mathrm{K}$ combined with four-factor prothrombin complex concentrate (4F-PCC) is the most rapid and effective regimen to restore hemostasis. For those on dabigatran, the highest quality data available for reversal are for idarucizumab, although it is not yet clear whether patients derive clinical benefit from this reversal. In the absence or failure of idarucizumab, activated prothrombin complex concentrate (aPCC) is recommended. For those on factor Xa inhibitors, the ideal reversal agent is not clear. Many providers use 4F-PCC or aPCC, but more specific agents are in clinical trials and may soon be available. In addition, the half-lives of the non-vitamin $\mathrm{K}$ antagonists are relatively short compared with warfarin, and so some patients may not have a clinically relevant coagulopathy at the time of presentation. Overall, the optimal reversal agent, when one is required, is a function of which anticoagulant the patient is taking.
\end{abstract}

In patients with acute intracerebral hemorrhage (ICH), one of the major concerns is ongoing bleeding or ICH expansion. ${ }^{1}$ Unfortunately, many with ICH are also anticoagulated at the time of presentation, often for prevention and treatment of thromboembolism. ${ }^{1}$ The patients anticoagulated with vitamin K antagonists (VKAs) or direct oral anticoagulants seem to be at higher risk of ongoing expansion and worse outcome. $^{2}$ As a result, clinical practice is typically focused on reversing their anticoagulant in the acute phase. It may be that rapid and effective reversal can reduce the risk of expansion and improve clinical outcome. ${ }^{3}$ A range of options is available, and the most effective strategy is a function of the agent the patient is taking and what tools are locally and emergently available.

\section{Warfarin}

Warfarin, a VKA, is a commonly prescribed anticoagulant for the prevention and treatment of thromboembolism. By inhibiting vitamin $\mathrm{K}$-dependent carboxylation and synthesis of coagulation factors II, VII, IX, and X, the coagulation cascade is impaired at multiple sites. This is evidenced by an elevation in the international normalized ratio (INR), which can increase a patient's risk of major and/or life-threatening bleeding including $\mathrm{ICH}^{4}$ Although warfarin-associated $\mathrm{ICH}$ can occur at a therapeutic INR of 2 to 3 , a supratherapeutic INR $>3$ has been associated with an even greater risk. ${ }^{5-7}$

Over the years, the incidence of warfarin-associated ICH has increased substantially, likely due to more widespread

Copyright $\odot 2017$ by Thieme Medical Publishers, Inc., 333 Seventh Avenue, New York, NY 10001, USA.

Tel: +1(212) 584-4662.
DOI https://doi.org/ 10.1055/s-0037-1607993. ISSN 1069-3424. 
use of warfarin..$^{8-10}$ Unfortunately, warfarin-associated ICH is associated with even higher risk of hematoma expansion than nonwarfarin ICH. ${ }^{11,12}$ Thus, prompt reversal of anticoagulation in warfarin-associated ICH is a crucial step with the goal of controlling bleeding and preventing further hematoma expansion. Several treatment modalities are available for reversing warfarin.

\section{Phytonadione (Vitamin K1)}

Vitamin K remains a mainstay of treatment for reversing the anticoagulant effects of warfarin. Administering vitamin $\mathrm{K}$ in a patient with adequate liver function promotes hepatic synthesis of coagulation factors II, VII, IX, and X, which can effectively normalize the INR. ${ }^{13}$ However, the reversal effects of vitamin $\mathrm{K}$ are not immediate, even when administered intravenously at high doses. The initial INR reduction seen with intravenous (IV) vitamin $\mathrm{K}$ takes place within the first 6 to 8 hours. ${ }^{14}$ This time course may not be rapid enough in patients with $\mathrm{ICH}$ since they are at risk of experiencing hematoma expansion early on after the initial bleed. ${ }^{15,16}$ Therefore, administering vitamin $\mathrm{K}$ monotherapy for emergent reversal in life-threatening ICH is not sufficient as it may be associated with a higher risk of hematoma growth when compared with the administration of coagulation factor products. ${ }^{17}$ Thus, it is recommended to administer vitamin $\mathrm{K}$ in combination with more rapid-acting coagulation factor products.

Regardless of the delayed onset of action, vitamin $\mathrm{K}$ provides a sustained reversal by restoring systemic levels of coagulation factors II, VII, IX, and X. IV administration of vitamin $\mathrm{K} 5$ to $10 \mathrm{mg}$ is the recommended route and dose in these situations. ${ }^{4,18}$ The infusion rate should not exceed $1 \mathrm{mg} /$ minute to minimize the risk of anaphylactoid reactions. Although rare, reactions have been reported to be severe enough to cause hemodynamic instability. ${ }^{19}$ To reduce the risk, many institutions dilute a 10 -mg IV vitamin $\mathrm{K}$ dose in 50 or $100 \mathrm{~mL}$ of $0.9 \%$ sodium chloride. In regard to other administration routes, oral possesses an onset time that is too long in emergent cases, subcutaneous is associated with erratic and unpredictable absorption, and intramuscular may cause bleeding and hematoma formation at the injection site. ${ }^{20-22}$

\section{Fresh-Frozen Plasma}

Prior to the widespread availability of concentrated coagulation factor products, fresh-frozen plasma (FFP) was administered at doses ranging from 10 to $20 \mathrm{~mL} / \mathrm{kg}$ for the reversal of warfarin-associated $\mathrm{ICH}^{23,24}$ This provides an exogenous source of all clotting factors and proteins found in blood to promote hemostasis. Unfortunately, FFP is associated with numerous risks including transfusion-related acute lung injury, infusion reactions, hypocalcemia, infectious complications, and transfusion-associated circulatory overload. ${ }^{25,26}$

The INR of FFP is estimated to be approximately 1.6; therefore, it is difficult for FFP transfusions alone to decrease a patient's INR to $\leq 1.5$; in addition, its administration takes several hours in standard clinical practice. ${ }^{27-29}$ Concomitant vitamin $\mathrm{K}$ administration is critical to eventually correct the
INR. In addition, FFP's utility may be further limited due to potential procurement delays (e.g., checking for blood compatibility and thawing). ${ }^{30,31}$

\section{Four-Factor Prothrombin Complex Concentrate}

Recently, four-factor prothrombin complex concentrate (4F-PCC), which contains a concentration of coagulation factors II, VII, IX, and X, has become available in the United States for rapid reversal of warfarin. Although 4FPCC has been available in Europe since 1996, this reversal agent was not approved for use in the United States until 2013. Commercially available as a lyophilized powder, 4FPCC can be quickly reconstituted and rapidly administered in small volumes. ${ }^{32}$ More importantly, 4F-PCC provides a faster time to INR reversal compared with FFP. ${ }^{33,34}$

Sarode et al analyzed 202 patients who were randomized to receive 25 to 50 units $/ \mathrm{kg}$ of $4 \mathrm{~F}-\mathrm{PCC}(n=98)$ or 10 to $15 \mathrm{~mL} / \mathrm{kg}$ of FFP $(n=104)$ for urgent VKA reversal in acute major bleeding. ${ }^{34}$ At 30 minutes after infusion, 4F-PCC was deemed superior to FFP in achieving an INR $\leq 1.3$ (62.2 vs. 9.6\%; difference, 52.6\%; 95\% confidence interval [CI], 39.4-65.9). In regard to patients who achieved effective hemostasis, 4F-PCC may have demonstrated a benefit over FFP for musculoskeletal and visible bleeds ( 82.6 vs. $50 \%$; difference, $32.6 \%$; $95 \% \mathrm{CI}$, 4.5-60.7), but was not statistically significantly superior to FFP for intracranial, gastrointestinal, and other nonvisible bleeds (69.3 vs. $71.1 \%$; difference, $1.7 \%$; $95 \% \mathrm{CI},-17.6$ to 14.2 ). However, only 24 patients (12 in each group) were treated for intracranial hemorrhage, which may not have provided sufficient power to detect a difference for this patient population.

Steiner et al performed a randomized trial comparing 30 units/kg of 4F-PCC ( $n=27)$ with $20 \mathrm{~mL} / \mathrm{kg}$ of FFP $(n=23)$ for VKA reversal specifically in patients with $\mathrm{ICH}^{3}$ At 3 hours after start of treatment, significantly more patients in the 4F-PCC group achieved an INR $\leq 1.2$ when compared with the FFP group (66.7 vs. $8.7 \%$; odds ratio [OR], 30.6; $95 \% \mathrm{CI}$, 4.7-197.9). Of note, patients in the FFP group who did not achieve INR correction at 3 hours subsequently received rescue treatment with $4 \mathrm{~F}-\mathrm{PCC}$. At 3 hours, mean hematoma expansion was lower in the $4 \mathrm{~F}-\mathrm{PCC}$ group (9.7 vs. $23.7 \mathrm{~mL}$; difference, $16.9 \mathrm{~mL}$; 95\% CI, 2.5-31.3). This difference was still present at 24 hours ( 8.3 vs. $22.1 \mathrm{~mL}$, difference, $16.4 \mathrm{~mL}$; 95\% CI, 2.9-29.9), despite the fact that most patients in the FFP group ended up receiving 4F-PCC as rescue treatment. There were no significant differences in 90-day mortality, functional independence, or quality of life scores; however, the trial was stopped early, and a low sample size may have limited the ability to detect an effect on outcome. Of note, five deaths due to hematoma expansion occurred within 48 hours of treatment; all five of these deaths occurred exclusively in the patients who received FFP initially.

Retrospective studies have also investigated VKA reversal specifically in the ICH population. Parry-Jones et al pooled data from an international registry and reported an equivalent adjusted risk of mortality in patients who received either a three-factor prothrombin complex concentrate (3F-PCC) or 4 F-PCC $(n=585)$ versus FFP $(n=377)$ alone. ${ }^{35}$ Interestingly, 
patients who received combination therapy with a PCC product and FFP $(n=131)$ trended toward a reduced adjusted risk of mortality, although this finding is limited by the small number of patients and the retrospective observational study design. Kuramatsu et al performed a multicenter retrospective cohort study of spontaneous $\mathrm{ICH}$ patients associated with VKA use $(n=1,176) .{ }^{36}$ Although a variety of reversal strategies were utilized, achievement of INR $<1.3$ within 4 hours was associated with lower rates of hematoma expansion (OR, 0.27; 95\% CI, 0.15-0.43). When combining that with systolic blood pressure $<160 \mathrm{~mm} \mathrm{Hg}$ within 4 hours, the rates of hematoma expansion were further reduced (OR, 0.17; 95\% CI, 0.11-0.33) Given that 4F-PCC corrects the coagulopathy more rapidly when compared with FFP, it serves as a more useful agent to achieve an INR $<1.3$ within 4 hours.

The package insert dosing of 4F-PCC for urgent VKA reversal recommends 25 to 50 units $/ \mathrm{kg}$ of factor IX (maximum weight of $100 \mathrm{~kg}$ ) based on the patient's body weight and INR. IV vitamin $\mathrm{K} 5$ to $10 \mathrm{mg}$ should be administered concomitantly to maintain coagulation factor levels to prevent a rebound INR elevation after the effects of 4F-PCC have diminished. ${ }^{37}$ There is currently no robust evidence regarding safety and effectiveness of repeat $4 \mathrm{~F}-\mathrm{PCC}$ dosing in the clinical setting. If the INR remains $\geq 1.4$ after $4 \mathrm{~F}-\mathrm{PCC}$ administration, expert opinion suggests further correction with FFP. $^{23}$

Many institutions have developed their own site-specific protocols for emergent VKA reversal. Results of a recent international survey of hospital protocols reported the use of fixed instead of variable dosing of 4F-PCC. Proposed benefits of fixed dosing protocols include cost savings and minimizing delays. ${ }^{38}$ Several studies demonstrated fixed doses of $1,000,1,500$, or 2,000 units to be effective at INR correction. $^{39-43}$ However, a recent study in patients with intracranial bleeding demonstrated a fixed dosing strategy of 1,000 units was not as effective as variable dosing in achieving an INR $\leq 1.5 .{ }^{44}$ Fixed weight-based doses of 25 and 30 units/kg have also been reported with effective INR correction. ${ }^{3,45}$ None of the aforementioned fixed dosing strategies has been validated in large clinical trials.

\section{Other Concentrated Coagulation Factor Products}

Prior to the era of widespread 4F-PCC availability, other concentrated coagulation factor products were investigated for urgent VKA reversal including 3F-PCC, activated prothrombin complex concentrate (aPCC), and recombinant activated factor VII (rFVIIa). None of these products is currently FDA approved for this indication.

In contrast to 4F-PCC, 3F-PCC contains limited amounts of factor VII. When compared with FFP and/or vitamin K, some observational studies suggested improved clinical outcomes in patients who received 3F-PCC. ${ }^{46-48}$ However, this comparison has never been performed in a randomized clinical trial. In recent years, a handful of retrospective studies including patients with intracranial bleeds have been published comparing 4F-PCC versus 3F-PCC with conflicting results. Three studies did find advantages of 4F-PCC over
3F-PCC. ${ }^{49-51}$ The most recently published study by Fischer et al reviewed 103 patients with intracranial bleeds on warfarin who received either PCC agent, with 52 of these patients presenting specifically with an $\mathrm{ICH}^{52}$ There were no statistical differences observed in any outcomes between either products. The higher quality of evidence supporting 4F-PCC for rapid INR reversal, combined with the fact that it contains therapeutic levels of factor VII, elevates 4F-PCC as the preferred agent over 3F-PCC in emergent and potentially life-threatening situations such as warfarin-associated $\mathrm{ICH}^{23}$

The evidence supporting aPCC in warfarin-associated ICH is limited. Wójcik et al performed a retrospective chart review of patients requiring reversal of warfarin for a lifethreatening bleed who were administered a fixed 500 or 1,000 unit dose of aPCC $(n=72)$ or FFP $(n=69) .{ }^{53}$ A total of 28 and 9 patients had intracranial bleeds in the aPCC and FFP groups, respectively. No significant differences in length of stay or mortality were noted. Yin et al conducted a retrospective analysis that included patients on warfarin therapy presenting with an intracranial bleed who received aPCC 15 to 25 units $/ \mathrm{kg}(n=28)$ or FFP $(n=50) .{ }^{54}$ The median time to an INR $<1.5$ was faster with aPCC: 0.5 versus 10 hours. At 24 hours, the achievement of INR correction was similar between the groups. No differences in mortality or other notable clinical outcomes were seen. Based on the available literature, aPCC seems to provide a more rapid INR correction when compared with FFP. However, there is a lack of robust data regarding efficacy and clinical outcomes and no randomized trials have been performed using aPCC. In addition, there has been concern of aPCC's thrombogenic risk especially at higher doses, likely due to the presence of activated factor VII. ${ }^{55}$

Interest in the use of rFVIIa was sparked from results of two randomized trials demonstrating that treatment with rFVIIa for a nonanticoagulant-associated ICH limits hematoma expansion, but did not significantly improve clinical outcomes. $^{56,57}$ To date, no randomized study has been performed to assess the effect of rFVIIa in warfarin-associated $\mathrm{ICH}$. In retrospective comparisons of warfarin-associated intracranial bleeds, rFVIIa has been shown to reverse the INR faster than FFP and 3F-PCC. ${ }^{58-62}$ However, there is no evidence suggesting there is a true clinical benefit of rFVIIa in terms of improving outcomes. There has also been concern with the significant thromboembolic rates traditionally associated with rFVIIa. ${ }^{63-65}$

Deloughery et al performed a retrospective analysis of patients on warfarin who received rFVIIa $(n=35)$, rFVIIa in combination with 3F-PCC $(n=102)$, or 4F-PCC $(n=58)$, with the majority of patients presenting with intracranial bleeds. ${ }^{66}$ All three groups achieved a mean postadministration INR $\leq 1.5$. The combination group was able to achieve a mean INR $\leq 1.3$ but was associated with a significantly higher incidence of deep vein thrombosis. This suggests a concerning thrombogenicity risk of administering multiple coagulation factor products concomitantly, especially if rFVIIa is part of the reversal regimen. The crude mortality rate and length of stay of survivors were lowest in the 4F-PCC group, 
although these differences did not reach statistical significance. Notably, patients who received rFVIIa alone required significantly more FFP transfusions. Another study also noted patients who received rFVIIa, despite achieving rapid INR correction, required more FFP transfusions when compared with other reversal strategies. ${ }^{67}$ It is important to note that although rFVIIa may correct the INR initially, physiologic hemostasis may remain acutely altered due to the lack of exogenous replenishment of coagulation factors II, IX, and X; the INR reversal may not reflect true correction of the coagulopathy. ${ }^{68}$ Woo et al reported incidences of INR rebound in patients who initially achieved INR correction after rFVIIa administration, despite the coadministration of IV vitamin $\mathrm{K}$, likely due to the short half-life of factor VIIa. ${ }^{59}$ For anticoagulant reversal in warfarin-induced ICH, rFVIIa is not currently recommended due to the lack of any convincing efficacy or outcomes data, concern for incomplete and inadequate reversal, and previous reports of significant thromboembolic rates. $^{23}$

\section{Conclusion}

Overall, for emergent reversal of warfarin in ICH, 4F-PCC plus vitamin $\mathrm{K}$ is the method that will most rapidly and reliably correct the coagulopathy. Although the evidence is inconclusive regarding whether a faster time to INR correction truly improves long-term outcomes, 4F-PCC may be associated with lower rates of hematoma expansion. This would be desirable since hematoma expansion is associated with mortality and poor functional outcome. ${ }^{69,70}$ After 4F-PCC and IV vitamin $\mathrm{K}$ are administered, patients should be monitored for the development of thromboembolic events. Reversal of anticoagulation may re-expose patients to their underlying prothrombotic disease state(s) and 4F-PCC itself may possess intrinsic procoagulant activity. ${ }^{71}$ Clinicians should also discuss when it would be safe to reinitiate anticoagulation, if indicated, after assessing the risk-versus-benefit profile.

\section{Direct Thrombin Inhibitors}

Dabigatran was approved by the FDA in 2010 as the first nonwarfarin oral anticoagulant to be introduced to the U.S. market. Dabigatran is a direct thrombin inhibitor that inhibits both free and fibrin-bound thrombins. Current indications include prevention and treatment of deep vein thrombosis and pulmonary embolism, nonvalvular atrial fibrillation, and postoperative thromboprophylaxis. One advantage of dabigatran is that the drug does not require routine laboratory monitoring. Dabigatran is associated with bleeding events such as $\mathrm{ICH}$, and the reported incidence of ICH is estimated at 0.24 to 0.8 per 100,000 patientdays. ${ }^{72,73}$ At the time of FDA approval, there was no antidote or known efficacious treatment for dabigatran-induced coagulopathy. As a result, attempts to restore hemostasis in case of emergencies involved a range of treatments including PCC, FFP, and rFVIla. The concept with PCC and FFP is that providing extrafactor II might override the effects of a factor II inhibitor, while use of rFVIIa might activate the coagulation cascade downstream of the factor II inhibition. Some advocated for aPCC to deliver "activated" coagulation factors. In 2015, the FDA approved idarucizumab, a monoclonal antibody fragment which binds specifically to dabigatran to neutralize the anticoagulant effect, for dabigatran reversal. ${ }^{74}$

\section{Coagulation Factor Delivery}

In 2011, Eerenberg et al published the results of a singlecenter, randomized, placebo-controlled, crossover study that evaluated the use of 4F-PCC (Cofact, Sanquin Blood Supply, Amsterdam, the Netherlands) to reverse the anticoagulant effect of dabigatran. ${ }^{75}$ Study authors enrolled 12 healthy male volunteers, who received $150 \mathrm{mg}$ of dabigatran twice daily for 2.5 days. On the third day, patients received an additional dose and then were admitted to the hospital for an infusion of 4F-PCC or placebo. Based on prior research, investigators chose a 4F-PCC dose of 50 units $/ \mathrm{kg}$. Activated partial thromboplastin time (aPTT), thrombin time (TT), and ecarin clotting time (ECT) were used to assess dabigatran anticoagulation. The trial found that 4F-PCC did not reverse the prolongation of aPTT, ECT, and TT due to dabigatran. No major or clinically relevant bleeding complications occurred during treatment.

A 2012 randomized, crossover study evaluated the effect of nonspecific reversal agents on the anticoagulant activity of dabigatran. ${ }^{76}$ Ten healthy, white, male volunteers, aged 18 to 45 years were administered a one-time dose of dabigatran $150 \mathrm{mg}$ orally. The three reversal agents tested were rFVIIa (Novoseven, NovoNordisk, Copenhagen, Denmark), aPCC (FEIBA, Baxter AG, Vienna, Austria), and 4F-PCC (Kanokad, LFB, Courtaboeuf, France), all at various concentrations. Thrombin generation tests including endogenous thrombin potential-area under the curve (ETP-AUC), maximum concentration of thrombin, lag time (LT), and time to reach maximum concentration of thrombin were measured. 4FPCC increased ETP-AUC, but had no effect on dabigatranmodified thrombin generation test kinetic parameters. rFVIIa at the higher dose and aPCC nearly completely corrected the altered LT. In summary, although 4F-PCC increased ETP, only rFVIIa and aPCC corrected thrombin activity.

Arellano-Rodrigo et al enrolled healthy volunteers to receive dabigatran $150 \mathrm{mg}$ twice daily for 5 days. ${ }^{77}$ Concentrations of rFVIIa equivalent to $270 \mu \mathrm{g} / \mathrm{kg}$, aPCC at 75 units $/ \mathrm{kg}$, and 4F-PCC (Beriplex, CSL Behring GmbH, Marburg, Germany) at 50 units/kg were added into blood samples. Dabigatran treatment significantly prolonged both PT and aPTT in blood samples drawn 2 to 3 hours after the last dose taken. While rFVIIa or aPCC partially improved all the parameters, 4F-PCC did not modify the prolonged aPTT observed after dabigatran treatment.

In one observational study, 14 patients from a prospective cohort were treated with aPCC for major bleeding. ${ }^{78}$ Patients were treated as per existing hospital protocol and were subsequently recruited for a 30-day follow-up. Effectiveness of aPCC was not evaluated by laboratory markers. Instead, the treating physician used an assessment guide, described in an article supplement, with outcomes defined as good, 
moderate, or poor/none for four different types of bleeding. Safety outcomes were arterial or venous thromboembolism (VTE) or death. A comparison was made with 28 historic cases of dabigatran-associated major bleeds treated with supportive care, by matching 1:2 for type of bleed, age, and sex. The effectiveness of aPCC was assessed as good in nine (64\%), moderate in five (36\%), and poor in none. There were no reported thromboembolic events and one death.

All three ex vivo studies enrolled healthy volunteers and tested against therapeutic levels of dabigatran. ${ }^{75-77}$ The studies attempted to use doses of factor similar to what would be used in actual patients but may not be generalizable from ex vivo extrapolation. Furthermore, correction of laboratory parameters may not translate into cessation of clinical bleeding. It is difficult to assess the risks of factor replacement in these small sample sizes. Historically, these treatments have been associated with thromboembolic complications. The only clinical trial had planned to enroll 32 patients in the aPCC arm but was stopped at 14 patients due to the availability of idarucizumab. ${ }^{78}$ Compared with historical controls with supportive care, it appears that aPCC administration was associated with more "good" and "moderate" outcomes with no reported thrombotic events, though statistical significance was not reached.

\section{Idarucizumab}

Idarucizumab was first studied in 110 healthy male volunteers aged 18 to 45 years to investigate pharmacokinetics, safety, and tolerability. ${ }^{79}$ The half-life of idarucizumab was determined to be 45 minutes with rapid peak and clearance. No effect on coagulation parameters or ETP was found in the absence of dabigatran. Adverse events were rare in both the idarucizumab and placebo groups. The same authors then completed a randomized, placebo-controlled, double-blind phase I study to assess the safety, tolerability, and efficacy of idarucizumab on the reversal of dabigatran-induced anticoagulation. ${ }^{80}$ Healthy, male volunteers aged 18 to 45 years were randomized sequentially into four idarucizumab dose groups $(1,2,4$, and $5 \mathrm{~g})$ or placebo. The diluted TT (dTT), ECT, TT, aPTT, and ETP were used to assess the anticoagulant effect of dabigatran. Forty-seven volunteers completed the study. Immediate and sustained reversal of dabigatran-associated increases in ECT, aPTT, and TT were noted with doses of idarucizumab of $2 \mathrm{~g}$ or more. Volunteers who received $1 \mathrm{~g}$ of idarucizumab did not have sustained reversal at 72 hours. Similar to prior studies, this trial did not measure the effect of idarucizumab on bleeding patients.

A prospective, phase 3 trial, called REVERSal Effects of Idarucizumab in Patients on Active Dabigatran (RE-VERSE $A D)$, evaluated patients on dabigatran with life-threatening bleeding (Group A) or requiring urgent surgery (Group B). ${ }^{81}$ An interim analysis was published in 2015, including 90 patients of the planned enrollment goal of $300 .^{82}$ The full RE-VERSE AD trial results were published in $2017 .{ }^{83}$ Five hundred three patients were ultimately enrolled, 301 in Group A and 202 in Group B. The primary outcome was maximum reversal of the anticoagulant effect of dabigatran within 4 hours after the administration of idarucizumab.
There was no control group. Ninety eight (32.6\%) patients in Group A had intracranial hemorrhage as the indication for reversal. Idarucizumab reversed laboratory markers of anticoagulation from dabigatran rapidly and completely, including dTT and ECT. It is important to note that not all institutions have these assays available. The authors reported almost universal and complete binding of dabigatran as evidence by minimal unbound dabigatran concentrations after idarucizumab; an effect that lasted at least 24 hours. The dose that appeared most effective is $5 \mathrm{~g}$ IV (two $2.5 \mathrm{~g}$ infusions given no more than 15 minutes apart). Nine patients received more than $5 \mathrm{~g}$ dose of idarucizumab. Ten percent of patients in the full cohort, compared with $25 \%$ in the interim analysis, had no laboratory evidence of dabigatran's presence prior to administration of idarucizumab (normal ECT and/or dTT), suggesting that many patients with dabigatran-associated ICH may not be truly anticoagulated at the time of presentation. Most concerning is the discrepancy between clinically relevant bleeding cessation times in the interim versus full analyses. In the interim analysis, it took 11.4 hours for cessation of bleeding compared with 2.5 hours in the full cohort. A closer look at the marked change is that it seems in the full analysis, $>55 \%$ of patients in Group A were excluded from time-to-bleeding assessment. Reasons for exclusion included no cessation of bleeding within 24 hours or the bleeding location could not be identified. This change in reporting suggests that the time-to-cessation results in the full cohort may be overly favorable. Surprisingly, most patients with ICH were excluded from any analysis of clinical hemostasis; oddly, serial CT scans were not performed or analyzed, and therefore, the authors did not evaluate the frequency with which ICH expansion occurred. Thrombotic events occurred in 24 patients (4.8\%) within 30 days after treatment; however, direct association of these events with idarucizumab administration is unclear.

Though idarucizumab reverses laboratory markers of dabigatran, it does not repair the damaged vessel. Patients with nonanticoagulated ICH often suffer hematoma expansion, and the best imaginable reversal agent can only convert anticoagulated to nonanticoagulated $\mathrm{ICH}^{84}$ Indeed, cases of sustained bleeding after idarucizumab have been reported. ${ }^{85,86}$ In addition, the approved $5 \mathrm{~g}$ dose may not be sufficient in all cases, particularly in patients with renal failure who are unable to clear dabigatran. ${ }^{87} \mathrm{~A}$ randomized, double-blind, crossover study evaluated 46 volunteers (12 aged 45-64 years, 16 aged 65-80 years, and 18 with mild or moderate renal impairment). ${ }^{88}$ All subjects received dabiga$\operatorname{tran} 220$ or $150 \mathrm{mg}$ twice daily for 4 days followed by varying doses of idarucizumab. Impaired renal function was associated with increased exposure and decreased clearance of idarucizumab. This may be expected as dabigatran is cleared more slowly with decreased renal function. Hemodialysis is effective in reducing dabigatran concentrations in patients with dabigatran-associated bleeding, though a rebound in dabigatran concentration may occur upon cessation of hemodialysis. ${ }^{89}$ Prolonged renal replacement therapy may be needed in some cases, but idarucizumab could also play a role. Importantly, rebound dabigatran concentrations have 
also been reported after idarucizumab administration in the setting of severe renal failure. ${ }^{90}$

\section{Conclusion}

Regarding dabigatran reversal, only PCC, rFVIIa, and idarucizumab have been studied in the format of clinical trials meeting methodology standards according to the GRADE (The Grading of Recommendations Assessment, Development and Evaluation) criteria. $^{91}$ All of the studies investigating PCC, aPCC, and rFVIIa were conducted in healthy volunteers and measured laboratory reversal of anticoagulation parameters. Activated PCC, and possibly rFVIIa, may be considered to reverse dabigatran in situations where potential benefit outweighs risk. Idarucizumab, however, is more specific, and rapidly reverses laboratory coagulation markers from dabigatran. Idarucizumab should be considered for emergent reversal of dabigatran-associated $\mathrm{ICH}$, though its clinical efficacy apart from laboratory parameter correction is still unclear. All of the clinical trials were sponsored by the antidote's pharmaceutical manufacturer, Boehringer Ingelheim, who also markets dabigatran. ${ }^{79-83,88}$

\section{Factor Xa Inhibitors}

Oral direct factor Xa (FXa) inhibitors were first approved by the FDA in 2011 with the introduction of rivaroxaban. Since then, apixaban, edoxaban, and betrixaban have also come to market in the United States. Multiple indications exist for these agents. While rivaroxaban, apixaban, and edoxaban are all indicated for primary stroke prevention in patients with nonvalvular atrial fibrillation and VTE treatment, rivaroxaban and apixaban are also approved for prevention of VTE in hip or knee replacement surgeries. ${ }^{92-94}$ Newly approved betrixaban is labeled for VTE prevention in hospitalized patients. ${ }^{95}$ Compared with warfarin, oral direct FXa inhibitors have lower major bleeding, ICH incidence, and mortality (10\% relative reduction) in patients with atrial fibrillation. ${ }^{96-98}$ As with dabigatran, the direct FXa inhibitors also carry the advantages of oral formulation, minimal food and drug interactions, and lack of necessary routine laboratory monitoring. However, unlike dabigatran, a FXa inhibitor-class specific reversal agent is not currently available in the United States. In clinical practice, providers use a range of strategies for reversal.

\section{Coagulation Factors}

Several animal and healthy human studies have investigated the use of PCC, aPCC, and rFVIIa in FXa inhibitor reversal. In their randomized, placebo-controlled, crossover study of 12 healthy male volunteers, Eerenberg et al found that rivaroxaban dosed $20 \mathrm{mg}$ twice daily significantly increased the prothrombin time (PT) and decreased the ETP. ${ }^{75}$ Both of these parameters were normalized after administration of 50 units/kg 4F-PCC (Cofact, Sanquin Blood Supply) and persisted through 24 hours. Compared with placebo, the PT reduction and ETP increase were statistically significant ( $p<0.001$ for both). However, both values nearly normalized at 24 hours with normal saline alone. In the 4F-PCC group, the rise in ETP over 24 hours to more than $100 \%$ baseline ETP suggested that there may have been an excess of thrombin generation. No bleeding complications or serious adverse events were reported.

Cheung et al addressed the PCC dosing concern with their single-center, randomized, double-blind, placebo-controlled crossover study. ${ }^{99}$ Six healthy male volunteers were administered apixaban $10 \mathrm{mg}$ twice daily for seven doses to reach steady state. Three hours after the last apixaban dose, subjects were randomized to receive 4F-PCC (Cofact, Sanquin Blood Supply) in doses of 25 or 37.5 units/kg or normal saline placebo. Subjects then underwent a 15- to 30-day washout period to resume apixaban and receive one of the other treatment regimens until all three sessions were complete. At 15 minutes after 4F-PCC administration, both doses increased ETP ( $p=0.06$ for 37.5 units $/ \mathrm{kg}$ and $p=0.03$ for 25 units $/ \mathrm{kg})$ and significantly decreased PT $(p<0.01$ for both). These effects were sustained at 24 hours compared with placebo. The only adverse reaction reported was transient calf numbness that had resolved by the time of report in one subject who received 37.5 units/kg 4F-PCC.

In their randomized, crossover, ex vivo study of 10 healthy male volunteers, Marlu et al also investigated rFVIIa (Novoseven, NovoNordisk) at doses equating 20,60, and 120 units/kg; aPCC (FEIBA, Baxter AG) at doses equating 20, 40, 80, and 160 units/kg; and 4F-PCC (Kanokad, LFB) at doses equating 12.5, 25, and 50 units/kg after a single dose of rivaroxaban $20 \mathrm{mg} .{ }^{76}$ Both 4F-PCC and aPCC, but not rFVIIa, affected quantitative parameters to return ETP-AUC to near baseline and correct thrombin peak. Kinetic parameters TTP and LT were best affected by rFVIIa and aPCC. In consideration of both the parameters examined, aPCC, at all doses, was the only agent that affected both quantitative (ETP-AUC, peak) and kinetic (TTP, LT) parameters. It is important to note that higher concentrations of 4FPCC and aPCC overcorrected the ETP-AUC from baseline and may suggest excess thrombin generation. These kinetic and quantitative parameter correction results were supported by an in vitro apixaban study comparing aPCC, 4F-PCC, and rFVIIa in healthy volunteer whole blood administered therapeutic or supratherapeutic apixaban concentrations. ${ }^{100}$ Although 4FPCC improved latency parameter PTonly and static parameters fibrin network structure, ETP, and peak height and rFVIIa decreased latency parameters PT, aPTT, LT, and thromboelastometry measured EXTEM and INTEM clotting time assays, aPCC improved all of these parameters in both apixaban concentrations.

Edoxaban reversal results were similar in a single-center, ex vivo [sic] study of six healthy volunteers. ${ }^{101}$ Two to four times supratherapeutic maximal concentrations of edoxaban were added to 15 whole blood sample aliquots per volunteer. After incubation, aPCC (FEIBA, Baxter AG) or rFVIIa (NovoSeven, Novo Nordisk) at concentrations corresponding to therapeutic doses of 50 and 100 and 40 and 90 units $/ \mathrm{kg}$, respectively, was added to the aliquots. Both aPCC and rFVIIa nearly normalized aPTT, PT, and extrinsic anti-FXa activity to baseline. Intrinsic FXa activity was slightly improved but not normalized.

Given the paucity of high-quality studies for coagulation factor use for ICH in patients on FXa inhibitors, either aPCC or 
$4 \mathrm{~F}-\mathrm{PCC}$ is reasonable to consider for these patients in the absence of FXa-specific antidotes. Activated and 4F-PCC doses between 25 and 50 units/ $\mathrm{kg}$ may be sufficient for FXa reversal as measured by kinetic and quantitative parameters. Based on potential for increased thrombin generation and coagulation risk, higher doses should be used with caution. Of note, current guidelines by the Neurocritical Care Society and Society of Critical Care Medicine recommend considering aPCC or 4F-PCC 50 units/kg use in patients on FXa inhibitors with $\mathrm{ICH}^{23}$

\section{Andexanet Alfa}

Andexanet alfa (Portola Pharmaceuticals, South San Francisco, CA) is a modified recombinant human FXa decoy protein that sequesters FXa inhibitors to restore endogenous FXa activity. In a rat model given various FXa inhibitors, including rivaroxaban, apixaban, and betrixaban, andexanet alfa infusion decreased INR, increased total plasma concentration, and decreased unbound drug. ${ }^{102}$ Initial randomized, double-blind, placebo-controlled, phase II studies validated dose-related efficacy on reducing anti-FXa activity in healthy volunteers given rivaroxaban, apixaban, and edoxaban for 6 days therapeutic regimens. ${ }^{103-105}$ It is currently in phase III clinical trials.

In two randomized, double-blind, placebo-controlled parallel trials of healthy volunteers, ANNEXA-A and ANNEXA-R evaluated adults aged 50 to 75 years assigned apixaban or rivaroxaban, respectively. ${ }^{106}$ After reaching steady-state plasma concentrations, subjects were given andexanet at peak plasma concentration as either bolus only (400 mg for apixaban or $800 \mathrm{mg}$ for rivaroxaban) or bolus plus a 120 minute infusion ( $4 \mathrm{mg} / \mathrm{min}$ for apixaban or $8 \mathrm{mg} / \mathrm{min}$ for rivaroxaban). Andexanet was able to decrease anti-FXa activity in both apixaban and rivaroxaban compared with placebo regardless of bolus and/or infusion regimen $(p<0.001)$. These effects persisted for up to 2 hours after andexanet administration. Secondary efficacy outcomes were also statistically significant in participants who received andexanet bolus with or without infusion with increased thrombin generation and decreased mean concentrations of unbound apixaban and rivaroxaban. No serious adverse events were reported, though andexanet recipients did have nonneutralizing antibody development (17\% compared with $2 \%$ placebo) and transient D-dimer and prothrombin fragment elevations.

After the initial study of healthy volunteers, an interim report of an ongoing multicenter, prospective, open-label, single-group study of patients with acute major bleeding was published. ${ }^{107}$ Andexanet alfa, a Novel Antidote to the Anticoagulation Effects of FXa Inhibitors (ANNEXA-4) investigated 67 patients with acute major bleeding who had received apixaban, rivaroxaban, edoxaban, or enoxaparin within 18 hours of enrollment. Major bleeding was defined as potentially life-threatening acute overt bleeding with signs of hemodynamic instability, acute overt bleeding with hemoglobin level $8 \mathrm{~g} / \mathrm{dL}$ or drop by $2 \mathrm{~g} / \mathrm{dL}$ from baseline, or acute symptomatic bleeding in a critical organ or area. Unlike REVERSE-AD, ANNEXA-4 did not include patients requiring urgent reversal for procedures. Eligible patients received andexanet as a bolus and 2 hours infusion in doses previously studied to produce anti-FXa activity reduction by at least $80 \%$. Of the patients included in this study, the mean age was 77 years, and all patients had either cardiovascular disease or thromboses. The oral FXa inhibitor patients were taking rivaroxaban $(48 \%, n=32)$ and apixaban $(46 \%$, $n=31$ ). Bleeding locations were gastrointestinal in 33 subjects (49\%), intracranial in 28 (42\%), and in other locations in 6 (9\%). The anti-FXa activity decreased in patients on rivaroxaban after both andexanet bolus (89\%, 95\% CI, 58-94\%) and infusion (86\%, 95\% CI, 55-93\%). Apixaban recipients also had anti-FXa activity reductions after both andexanet bolus (93\%, 95\% CI, 87-94\%) and infusion (92\%, 95\% CI, 85-94\%). Clinically, excellent or good hemostasis was achieved at 12 hours postinfusion in 79\% (31 excellent and 6 good) of the efficacy population. Nine patients had poor or no hemostasis 12 hours after andexanet infusion. As there was no control group, it is unclear how much hemostasis was improved over placebo. Compared with the healthy volunteer groups of ANNEXA-A and ANNEXA-R, the postinfusion 2-hour duration of anti-FXa activity reversal was consistent.

At 4 hours postinfusion, anti-FXa activity reversal decreased to the expected anticoagulant clearance rate (the activity level expected of placebo treated patients). In other words, the andexanet effect lasts from bolus through infusion, then likely disappears by 4 hours after the end of infusion. It may be that this is the most critical time frame for anticoagulant reversal, but it is difficult to confirm this absent a control group.

Unlike the ANNEXA-A and ANNEXA-R trials, patients did not experience any infusion-related reactions or antibody development. However, $18 \%(n=12)$ did have thrombotic events that included seven deep vein thromboses, five strokes, one pulmonary embolism, and one myocardial infarction. Most safety events occurred between follow-up days 4 and 30. This event rate is high compared with other studies of thromboembolism after anticoagulation reversal. It is not clear whether this is due to an effect of acute anticoagulation reversal or the natural course of disease in this critically ill patient population. While the ANNEXA-4 trial has shown promising results for andexanet alfa use in actively bleeding patients, this study was a single arm treatment that was not compared with any other current standards of treatment, such as FFP or coagulation factors. It is also unknown whether faster andexanet administration would affect outcomes, as the average time to andexanet was $4.8 \pm 1.9$ hours. In addition, this study did not include patients on edoxaban nor enoxaparin in their interim analyses; betrixaban was not FDA approved during this study period. Andexanet is not yet FDA approved, and the trial is ongoing. 108

There are currently no human studies comparing andexanet alfa with coagulation factors. However, in a liver laceration animal model by Lu et al, rivaroxaban-anticoagulated rabbits that received andexanet alfa had significantly less blood loss ( $p=0.004)$ and rapid reduction of anti-FXa activity and unbound rivaroxaban concentrations. The rabbits 
that received 3F-PCC and 4F-PCC did not have reduction of any of these parameters. ${ }^{109}$

\section{Ciraparantag}

Ciraparantag (PER977, Perosphere, Danbury, CT) is a small molecule that hydrogen binds noncovalently and inhibits target site binding of multiple anticoagulants, including FXa inhibitors, direct thrombin inhibitors, and heparins. In a preclinical in vitro study comparing ciraparantag with andexanet alfa, ciraparantag did not reverse FXa activity, unlike andexanet which had dose-dependent effects. In addition, factors $\mathrm{X}$ and IX activity, aPTT, and thrombin generation were potentiated by ciraparantag, suggesting potential procoagulant activity. ${ }^{110}$ A randomized, double-blind, placebo-controlled, safety, tolerability, pharmacokinetic, and pharmacodynamic trial examined ciraparantag administration after single-dose edoxaban compared with ciraparantag alone. ${ }^{111}$ Doses ranging from 100 to $300 \mathrm{mg}$ restored fibrin diameters to baseline levels 30 minutes after ciraparantag administration. There was no evidence of procoagulant activity. Further information is expected with the placebo-controlled, single-blind study of rivaroxaban reversal with ciraparantag that is actively recruiting. $^{112}$

\section{Conclusion}

Current available studies suggest andexanet alfa has the strongest evidence for oral FXa inhibitor reversal. However, andexanet has not been studied with a comparator in humans and is not yet clinically available. Coagulation factors and ciraparantag have only been studied in animal models and ex vivo healthy humans. Although it is not yet clear what currently available agent (if any) effectively reverses FXa inhibitors, current guidelines recommend coagulation factors aPCC or 4F-PCC as the agents with the most available data. ${ }^{23}$ PCC doses between 25 and 50 units/kg may be sufficient for FXa reversal as measured by kinetic and quantitative parameters.

\section{Conclusion}

Overall, anticoagulants provide tremendous benefits in prevention of stroke and other forms of thromboembolism. However, when patients develop ICH while anticoagulated, emergency reversal is typically provided in hopes of limiting bleeding and improving outcome. For those on VKAs such as warfarin, IV vitamin $\mathrm{K}$ with 4F-PCC appears to provide the most rapid and effective reversal. For those taking factor IIa inhibitors (dabigatran), IV idarucizumab is the most specific reversal agent available. aPCC, and possibly rFVIIa, is also alternatives (or adjuncts in some cases). For those taking FXa inhibitors (rivaroxaban, apixaban, edoxaban, and betrixaban), the optimal agent is not currently clear. Current guidelines recommend 4F-PCC (which contains factor X), and possibly aPCC, as the agents with the most supportive data. If andexanet alfa becomes available, it will likely supplant 4F-PCC, as it is more specific for anti-FXa activity. Ciraparantag may become a more universal reversal agent but is at earlier stages of testing.

\section{References}

1 Morotti A, Goldstein JN. Diagnosis and management of acute intracerebral hemorrhage. Emerg Med Clin North Am 2016;34 (04):883-899

2 Morotti A, Goldstein JN. New oral anticoagulants and their reversal agents. Curr Treat Options Neurol 2016;18(11):47

3 Steiner T, Poli S, Griebe M, et al. Fresh frozen plasma versus prothrombin complex concentrate in patients with intracranial haemorrhage related to vitamin $\mathrm{K}$ antagonists (INCH): a randomised trial. Lancet Neurol 2016;15(06):566-573

4 Holbrook A, Schulman S, Witt DM, et al. Evidence-based management of anticoagulant therapy: antithrombotic therapy and prevention of thrombosis, 9th ed: American College of Chest Physicians Evidence-Based Clinical Practice Guidelines. Chest 2012;141(2, Suppl):e152S-e184S

5 García-Rodríguez LA, Gaist D, Morton J, Cookson C, GonzálezPérez A. Antithrombotic drugs and risk of hemorrhagic stroke in the general population. Neurology 2013;81(06):566-574

6 Hart RG, Tonarelli SB, Pearce LA. Avoiding central nervous system bleeding during antithrombotic therapy: recent data and ideas. Stroke 2005;36(07):1588-1593

7 Rosand J, Eckman MH, Knudsen KA, Singer DE, Greenberg SM. The effect of warfarin and intensity of anticoagulation on outcome of intracerebral hemorrhage. Arch Intern Med 2004;164 (08):880-884

8 Ikram MA, Wieberdink RG, Koudstaal PJ. International epidemiology of intracerebral hemorrhage. Curr Atheroscler Rep 2012;14(04):300-306

9 Huhtakangas J, Tetri S, Juvela S, Saloheimo P, Bode MK, Hillbom M. Effect of increased warfarin use on warfarin-related cerebral hemorrhage: a longitudinal population-based study. Stroke 2011;42(09):2431-2435

10 Flaherty ML, Kissela B, Woo D, et al. The increasing incidence of anticoagulant-associated intracerebral hemorrhage. Neurology 2007;68(02):116-121

11 Cucchiara B, Messe S, Sansing L, Kasner S, Lyden P; CHANT Investigators. Hematoma growth in oral anticoagulant related intracerebral hemorrhage. Stroke 2008;39(11):2993-2996

12 Flibotte JJ, Hagan N, O'Donnell J, Greenberg SM, Rosand J. Warfarin, hematoma expansion, and outcome of intracerebral hemorrhage. Neurology 2004;63(06):1059-1064

13 Dentali F, Ageno W, Crowther M. Treatment of coumarin-associated coagulopathy: a systematic review and proposed treatment algorithms. J Thromb Haemost 2006;4(09):1853-1863

14 Kalus JS. Pharmacologic interventions for reversing the effects of oral anticoagulants. Am J Health Syst Pharm 2013;70(10, Suppl 1):S12-S21

15 Brott T, Broderick J, Kothari R, et al. Early hemorrhage growth in patients with intracerebral hemorrhage. Stroke 1997;28(01): $1-5$

16 Kazui S, Naritomi H, Yamamoto H, Sawada T, Yamaguchi T. Enlargement of spontaneous intracerebral hemorrhage. Incidence and time course. Stroke 1996;27(10):1783-1787

17 Huttner HB, Schellinger PD, Hartmann M, et al. Hematoma growth and outcome in treated neurocritical care patients with intracerebral hemorrhage related to oral anticoagulant therapy: comparison of acute treatment strategies using vitamin $\mathrm{K}$, fresh frozen plasma, and prothrombin complex concentrates. Stroke 2006;37(06):1465-1470

18 Hemphill JC III, Greenberg SM, Anderson CS, et al; American Heart Association Stroke Council; Council on Cardiovascular and Stroke Nursing; Council on Clinical Cardiology. Guidelines for the management of spontaneous intracerebral hemorrhage: a guideline for healthcare professionals from the American Heart Association/ American Stroke Association. Stroke 2015;46(07):2032-2060

19 Britt RB, Brown JN. Characterizing the severe reactions of parenteral vitamin K1. Clin Appl Thromb Hemost 2016: 1076029616674825 
20 Lubetsky A, Yonath H, Olchovsky D, Loebstein R, Halkin H, Ezra D. Comparison of oral vs intravenous phytonadione (vitamin K1) in patients with excessive anticoagulation: a prospective randomized controlled study. Arch Intern Med 2003;163(20):2469-2473

21 Crowther MA, Douketis JD, Schnurr T, et al. Oral vitamin K lowers the international normalized ratio more rapidly than subcutaneous vitamin $\mathrm{K}$ in the treatment of warfarin-associated coagulopathy. A randomized, controlled trial. Ann Intern Med 2002; 137(04):251-254

22 Watson HG, Baglin T, Laidlaw SL, Makris M, Preston FE. A comparison of the efficacy and rate of response to oral and intravenous vitamin $\mathrm{K}$ in reversal of over-anticoagulation with warfarin. Br J Haematol 2001;115(01):145-149

23 Frontera JA, Lewin JJ III, Rabinstein AA, et al. Guideline for reversal of antithrombotics in intracranial hemorrhage: a statement for healthcare professionals from the Neurocritical Care Society and Society of Critical Care Medicine. Neurocrit Care 2016;24(01):6-46

24 Brophy GM, Human T, Shutter L. Emergency neurological life support: pharmacotherapy. Neurocrit Care 2015;23(Suppl 2): S48-S68

25 Pandey S, Vyas GN. Adverse effects of plasma transfusion. Transfusion 2012;52(Suppl 1):65S-79S

26 Marshall AL, Levine M, Howell ML, et al. Dose-associated pulmonary complication rates after fresh frozen plasma administration for warfarin reversal. J Thromb Haemost 2016;14(02): 324-330

27 Yazer MH. The how's and why's of evidence based plasma therapy. Korean J Hematol 2010;45(03):152-157

28 Abdel-Wahab OI, Healy B, Dzik WH. Effect of fresh-frozen plasma transfusion on prothrombin time and bleeding in patients with mild coagulation abnormalities. Transfusion 2006;46(08): 1279-1285

29 Holland LL, Brooks JP. Toward rational fresh frozen plasma transfusion: the effect of plasma transfusion on coagulation test results. Am J Clin Pathol 2006;126(01):133-139

30 Lee SB, Manno EM, Layton KF, Wijdicks EF. Progression of warfarin-associated intracerebral hemorrhage after INR normalization with FFP. Neurology 2006;67(07):1272-1274

31 Goldstein JN, Thomas SH, Frontiero V, et al. Timing of fresh frozen plasma administration and rapid correction of coagulopathy in warfarin-related intracerebral hemorrhage. Stroke 2006;37(01): 151-155

32 Cada DJ, Levien TL, Baker DE. Prothrombin complex concentrate. Hosp Pharm 2013;48(11):951-957

33 Goldstein JN, Refaai MA, Milling TJ Jr, et al. Four-factor prothrombin complex concentrate versus plasma for rapid vitamin $\mathrm{K}$ antagonist reversal in patients needing urgent surgical or invasive interventions: a phase $3 \mathrm{~b}$, open-label, non-inferiority, randomised trial. Lancet 2015;385(9982):2077-2087

34 Sarode R, Milling TJ Jr, Refaai MA, et al. Efficacy and safety of a 4-factor prothrombin complex concentrate in patients on vitamin $\mathrm{K}$ antagonists presenting with major bleeding: a randomized, plasma-controlled, phase IIIb study. Circulation 2013;128(11): 1234-1243

35 Parry-Jones AR, Di Napoli M, Goldstein JN, et al. Reversal strategies for vitamin $\mathrm{K}$ antagonists in acute intracerebral hemorrhage. Ann Neurol 2015;78(01):54-62

36 Kuramatsu JB, Gerner ST, Schellinger PD, et al. Anticoagulant reversal, blood pressure levels, and anticoagulant resumption in patients with anticoagulation-related intracerebral hemorrhage. JAMA 2015;313(08):824-836

$37 \mathrm{Sin} \mathrm{JH}$, Berger K, Lesch CA. Four-factor prothrombin complex concentrate for life-threatening bleeds or emergent surgery: a retrospective evaluation. J Crit Care 2016;36:166-172

38 Gorlin J, Kinney S, Fung MK, Tinmouth A; BEST Collaborative. Prothrombin complex concentrate for emergent reversal of warfarin: an international survey of hospital protocols. Vox Sang 2017;112(06):595-597

39 Klein L, Peters J, Miner J, Gorlin J. Evaluation of fixed dose 4-factor prothrombin complex concentrate for emergent warfarin reversal. Am J Emerg Med 2015;33(09):1213-1218

40 Hirri HM, Green PJ. Audit of warfarin reversal using a new Octaplex reduced dose protocol. Transfus Apheresis Sci 2014; 51(02):141-145

41 Khorsand N, Veeger NJ, van Hest RM, Ypma PF, Heidt J, Meijer K. An observational, prospective, two-cohort comparison of a fixed versus variable dosing strategy of prothrombin complex concentrate to counteract vitamin $\mathrm{K}$ antagonists in 240 bleeding emergencies. Haematologica 2012;97(10):1501-1506

42 Khorsand N, Veeger NJ, Muller M, et al. Fixed versus variable dose of prothrombin complex concentrate for counteracting vitamin K antagonist therapy. Transfus Med 2011;21(02):116-123

43 Varga C, Al-Touri S, Papadoukakis S, Caplan S, Kahn S, Blostein M. The effectiveness and safety of fixed low-dose prothrombin complex concentrates in patients requiring urgent reversal of warfarin. Transfusion 2013;53(07):1451-1458, quiz 1450

44 Abdoellakhan RA, Miah IP, Khorsand N, Meijer K, Jellema K. Fixed versus variable dosing of prothrombin complex concentrate in vitamin $\mathrm{K}$ antagonist-related intracranial hemorrhage: a retrospective analysis. Neurocrit Care 2017;26(01):64-69

45 Appleby N, Groarke E, Crowley M, et al. Reversal of warfarin anticoagulation using prothrombin complex concentrate at 25 IU $\mathrm{kg}(-1)$ : results of the RAPID study. Transfus Med 2017;27(01): 66-71

46 Frontera JA, Gordon E, Zach V, et al. Reversal of coagulopathy using prothrombin complex concentrates is associated with improved outcome compared to fresh frozen plasma in warfarin-associated intracranial hemorrhage. Neurocrit Care 2014; 21(03):397-406

47 Hanger HC, Geddes JA, Wilkinson TJ, Lee M, Baker AE. Warfarinrelated intracerebral haemorrhage: better outcomes when reversal includes prothrombin complex concentrates. Intern Med J 2013;43(03):308-316

48 Kuwashiro T, Yasaka M, Itabashi R, et al. Effect of prothrombin complex concentrate on hematoma enlargement and clinical outcome in patients with anticoagulant-associated intracerebral hemorrhage. Cerebrovasc Dis 2011;31(02):170-176

49 Jones GM, Erdman MJ, Smetana KS, Mohrien KM, Vandigo JE, Elijovich L. 3-factor versus 4-factor prothrombin complex concentrate for warfarin reversal in severe bleeding: a multicenter, retrospective, propensity-matched pilot study. J Thromb Thrombolysis 2016;42(01):19-26

50 Al-Majzoub O, Rybak E, Reardon DP, Krause P, Connors JM. Evaluation of warfarin reversal with 4-factor prothrombin complex concentrate compared to 3-factor prothrombin complex concentrate at a Tertiary Academic Medical Center. J Emerg Med 2016;50(01):7-13

51 Voils SA, Holder MC, Premraj S, Catlin JR, Allen BR. Comparative effectiveness of 3- versus 4-factor prothrombin complex concentrate for emergent warfarin reversal. Thromb Res 2015;136 (03):595-598

52 Fischer D, Sorensen J, Fontaine GV. Three-factor versus four-factor prothrombin complex concentrate for the emergent management of warfarin-associated intracranial hemorrhage. Neurocrit Care 2017. Doi: $10.1007 / \mathrm{s} 12028-017-0374-y$

53 Wójcik C, Schymik ML, Cure EG. Activated prothrombin complex concentrate factor VIII inhibitor bypassing activity (FEIBA) for the reversal of warfarin-induced coagulopathy. Int J Emerg Med 2009;2(04):217-225

54 Yin EB, Tan B, Nguyen T, et al. Safety and effectiveness of factor VIII inhibitor bypassing activity (FEIBA) and fresh frozen plasma in oral anticoagulant-associated intracranial hemorrhage: a retrospective analysis. Neurocrit Care 2017;27(01):51-59 
55 Aledort LM. Factor VIII inhibitor bypassing activity (FEIBA) addressing safety issues. Haemophilia 2008;14(01):39-43

56 Mayer SA, Brun NC, Begtrup K, et al; Recombinant Activated Factor VII Intracerebral Hemorrhage Trial Investigators. Recombinant activated factor VII for acute intracerebral hemorrhage. N Engl J Med 2005;352(08):777-785

57 Mayer SA, Brun NC, Begtrup K, et al; FAST Trial Investigators. Efficacy and safety of recombinant activated factor VII for acute intracerebral hemorrhage. N Engl J Med 2008;358(20): 2127-2137

58 Chapman SA, Irwin ED, Abou-Karam NM, et al. Comparison of 3factor prothrombin complex concentrate and low-dose recombinant factor VIIa for warfarin reversal. World J Emerg Surg 2014;9:27

59 Woo CH, Patel N, Conell C, et al. Rapid warfarin reversal in the setting of intracranial hemorrhage: a comparison of plasma, recombinant activated factor VII, and prothrombin complex concentrate. World Neurosurg 2014;81(01):110-115

60 Pinner NA, Hurdle AC, Oliphant C, Reaves A, Lobo B, Sills A. Treatment of warfarin-related intracranial hemorrhage: a comparison of prothrombin complex concentrate and recombinant activated factor VII. World Neurosurg 2010;74(06):631-635

61 Ilyas C, Beyer GM, Dutton RP, Scalea TM, Hess JR. Recombinant factor VIIa for warfarin-associated intracranial bleeding. J Clin Anesth 2008;20(04):276-279

62 Brody DL, Aiyagari V, Shackleford AM, Diringer MN. Use of recombinant factor VIIa in patients with warfarin-associated intracranial hemorrhage. Neurocrit Care 2005;2(03):263-267

63 O'Connell KA, Wood JJ, Wise RP, Lozier JN, Braun MM. Thromboembolic adverse events after use of recombinant human coagulation factor VIIa. JAMA 2006;295(03):293-298

64 Aledort LM. Comparative thrombotic event incidence after infusion of recombinant factor VIIa versus factor VIII inhibitor bypass activity. J Thromb Haemost 2004;2(10):1700-1708

65 Diringer MN, Skolnick BE, Mayer SA, et al. Thromboembolic events with recombinant activated factor VII in spontaneous intracerebral hemorrhage: results from the Factor Seven for Acute Hemorrhagic Stroke (FAST) trial. Stroke 2010;41(01): 48-53

66 DeLoughery E, Avery B, DeLoughery TG. Retrospective study of rFVIIa, 4-factor PCC, and a rFVIIa and 3-factor PCC combination in improving bleeding outcomes in the warfarin and non-warfarin patient. Am J Hematol 2016;91(07):705-708

67 Nishijima DK, Dager WE, Schrot RJ, Holmes JF. The efficacy of factor VIla in emergency department patients with warfarin use and traumatic intracranial hemorrhage. Acad Emerg Med 2010; 17(03):244-251

68 Skolnick BE, Mathews DR, Khutoryansky NM, Pusateri AE, Carr ME. Exploratory study on the reversal of warfarin with rFVIIa in healthy subjects. Blood 2010;116(05):693-701

69 Dowlatshahi D, Demchuk AM, Flaherty ML, Ali M, Lyden PL, Smith EE; VISTA Collaboration. Defining hematoma expansion in intracerebral hemorrhage: relationship with patient outcomes. Neurology 2011;76(14):1238-1244

70 Davis SM, Broderick J, Hennerici M, et al; Recombinant Activated Factor VII Intracerebral Hemorrhage Trial Investigators. Hematoma growth is a determinant of mortality and poor outcome after intracerebral hemorrhage. Neurology 2006;66 (08):1175-1181

71 Sørensen B, Spahn DR, Innerhofer P, Spannagl M, Rossaint R. Clinical review: prothrombin complex concentrates-evaluation of safety and thrombogenicity. Crit Care 2011;15(01):201

72 Southworth MR, Reichman ME, Unger EF. Dabigatran and postmarketing reports of bleeding. N Engl J Med 2013;368(14): $1272-1274$

73 Graham DJ, Reichman ME, Wernecke M, et al. Cardiovascular, bleeding, and mortality risks in elderly Medicare patients trea- ted with dabigatran or warfarin for nonvalvular atrial fibrillation. Circulation 2015;131(02):157-164

74 Schiele F, van Ryn J, Canada K, et al. A specific antidote for dabigatran: functional and structural characterization. Blood 2013;121(18):3554-3562

75 Eerenberg ES, Kamphuisen PW, Sijpkens MK, Meijers JC, Buller HR, Levi M. Reversal of rivaroxaban and dabigatran by prothrombin complex concentrate: a randomized, placebo-controlled, crossover study in healthy subjects. Circulation 2011;124(14): 1573-1579

76 Marlu R, Hodaj E, Paris A, Albaladejo P, Cracowski JL, Pernod G. Effect of non-specific reversal agents on anticoagulant activity of dabigatran and rivaroxaban: a randomised crossover ex vivo study in healthy volunteers. Thromb Haemost 2012;108(02): 217-224

77 Arellano-Rodrigo E, Lopez-Vilchez I, Galan AM, et al. Coagulation factor concentrates fail to restore alterations in fibrin formation caused by rivaroxaban or dabigatran in studies with flowing blood from treated healthy volunteers. Transfus Med Rev 2015; 29(04):242-249

78 Schulman S, Ritchie B, Nahirniak S, et al; Study investigators. Reversal of dabigatran-associated major bleeding with activated prothrombin concentrate: a prospective cohort study. Thromb Res 2017;152:44-48

79 Glund S, Moschetti V, Norris S, et al. A randomised study in healthy volunteers to investigate the safety, tolerability and pharmacokinetics of idarucizumab, a specific antidote to dabigatran. Thromb Haemost 2015;113(05):943-951

80 Glund S, Stangier J, Schmohl M, et al. Safety, tolerability, and efficacy of idarucizumab for the reversal of the anticoagulant effect of dabigatran in healthy male volunteers: a randomised, placebo-controlled, double-blind phase 1 trial. Lancet 2015;386 (9994):680-690

81 Pollack CV Jr, Reilly PA, Bernstein R, et al. Design and rationale for RE-VERSE AD: a phase 3 study of idarucizumab, a specific reversal agent for dabigatran. Thromb Haemost 2015;114(01): 198-205

82 Pollack CV Jr, Reilly PA, Eikelboom J, et al. Idarucizumab for dabigatran reversal. N Engl J Med 2015;373(06):511-520

83 Pollack CV Jr, Reilly PA, van Ryn J, et al. Idarucizumab for dabigatran reversal - full cohort analysis. N Engl J Med 2017; 377(05):431-441

84 Brouwers HB, Greenberg SM. Hematoma expansion following acute intracerebral hemorrhage. Cerebrovasc Dis 2013;35(03): 195-201

85 Alhashem HM, Avendano C, Hayes BD, Winters ME. Persistent life-threatening hemorrhage after administration of idarucizumab. Am J Emerg Med 2017;35(01):193.e3-193.e5

86 Steele AP, Lee JA, Dager WE. Incomplete dabigatran reversal with idarucizumab. Clin Toxicol (Phila) 2017:1-3

87 Simon A, Domanovits H, Ay C, Sengoelge G, Levy JH, Spiel AO. The recommended dose of idarucizumab may not always be sufficient for sustained reversal of dabigatran. J Thromb Haemost 2017;15(07):1317-1321

88 Glund S, Stangier J, van Ryn J, et al. Effect of age and renal function on idarucizumab pharmacokinetics and idarucizumabmediated reversal of dabigatran anticoagulant activity in a randomized, double-blind, crossover phase Ib study. Clin Pharmacokinet 2017;56(01):41-54

89 Chai-Adisaksopha C, Hillis C, Lim W, Boonyawat K, Moffat K, Crowther M. Hemodialysis for the treatment of dabigatranassociated bleeding: a case report and systematic review. J Thromb Haemost 2015;13(10):1790-1798

90 Stecher A, Vene N, Mavri A, Mijovski MB, Krevel B, Gradišek P. Late rebound of dabigatran levels after idarucizumab reversal in two patients with severe renal failure. Eur J Anaesthesiol 2017; 34(06):400-402 
91 Guyatt GH, Oxman AD, Vist GE, et al; GRADE Working Group. GRADE: an emerging consensus on rating quality of evidence and strength of recommendations. BMJ 2008;336(7650):924-926

92 Xarelto (R) [package insert]. Titusville, NJ: Janssen Pharmaceuticals, Inc.; 2017

93 Eliquis (R) [package insert]. Princeton, NJ: Bristol-Myers Squibb Company; 2016

94 Savaysa (R) [package insert]. Parsippany, NJ: Daiichi Sankyo, Inc.; 2016

95 Bevyxxa (R) [package insert]. South San Francisco, CA: Portola Pharmaceuticals, Inc; 2017

96 Robertson L, Kesteven P, McCaslin JE. Oral direct thrombin inhibitors or oral factor Xa inhibitors for the treatment of pulmonary embolism. Cochrane Database Syst Rev 2015;(12): CD010957

97 Held C, Hylek EM, Alexander JH, et al. Clinical outcomes and management associated with major bleeding in patients with atrial fibrillation treated with apixaban or warfarin: insights from the ARISTOTLE trial. Eur Heart J 2015;36(20):1264-1272

98 Bruins Slot KM, Berge E. Factor Xa inhibitors versus vitamin $\mathrm{K}$ antagonists for preventing cerebral or systemic embolism in patients with atrial fibrillation. Cochrane Database Syst Rev 2013;(08):CD008980

99 Cheung YW, Barco S, Hutten BA, Meijers JC, Middeldorp S, Coppens $M$. In vivo increase in thrombin generation by fourfactor prothrombin complex concentrate in apixaban-treated healthy volunteers. J Thromb Haemost 2015;13(10):1799-1805

100 Martin A-C, Gouin-Thibault I, Siguret V, et al. Multimodal assessment of non-specific hemostatic agents for apixaban reversal. J Thromb Haemost 2015;13(03):426-436

101 Halim A-B, Samama MM, Mendell J. Ex vivo reversal of the anticoagulant effects of edoxaban. Thromb Res 2014;134(04): 909-913

102 Lu G, DeGuzman FR, Hollenbach SJ, et al. A specific antidote for reversal of anticoagulation by direct and indirect inhibitors of coagulation factor Xa. Nat Med 2013;19(04):446-451

103 Crowther M, Levy GG, Lu G, et al. A phase 2 randomized, doubleblind, placebo-controlled trial demonstrating reversal of edoxaban-induced anticoagulation in healthy subjects by andexanet alfa (PRT064445), a universal antidote for factor Xa (fXa) inhibitors. Blood 2013;124:4269

104 Crowther M, Vandana M, Michael K, et al. A phase 2 randomized, double-blind, placebo-controlled trial demonstrating reversal of rivaroxaban-induced anticoagulation in healthy subjects by andexanet alfa (PRT064445), an antidote for FXa inhibitors. Blood 2013;122:3636

105 Crowther M, Lu G, Conley P, et al. Sustained reversal of apixaban anticoagulation with andexanet alfa using a bolus plus infusion regimen in a phase 2 placebo controlled trial. Eur Heart J 2014;35 (Suppl 1):137

106 Siegal DM, Curnutte JT, Connolly SJ, et al. Andexanet alfa for the reversal of factor Xa inhibitor activity. N Engl J Med 2015;373 (25):2413-2424

107 Connolly SJ, Milling TJ Jr, Eikelboom JW, et al; ANNEXA-4 Investigators. Andexanet alfa for acute major bleeding associated with factor Xa inhibitors. N Engl J Med 2016;375(12):1131-1141

108 News Release: Portola Pharmaceutical Receives Complete Response Letter from FDA for Biologics License Application for AndexXa ${ }^{\mathrm{TM}}$ (andexanet alfa). Portola Pharmaceuticals, Inc. Available at: http://investors.portola.com/phoenix.zhtml?c=198136\& $\mathrm{p}=$ irol-newsroomArticle\&ID=2196085. Updated August 18, 2016. Accessed July 5, 2017

109 Lu G, Pine P, DeGuzman F, et al. Reversal of anticoagulation effects of rivaroxaban and associated bleeding in a rabbit acute hemorrhage model by andexanet alfa vs. coagulation replacement factors. Neurology 2017;88(16 Supplement P5.054): 1526-1632

110 Lu G, Kotha J, Cardenas JM, et al. In vitro characterization of andexanet alfa (PRT064445), a specific fXa inhibitor antidote versus aripazine (PER977), a non-specific reversal agent. Circulation 2014;130:A18218

111 Ansell JE, Bakhru SH, Laulicht BE, et al. Single-dose ciraparantag safely and completely reverses anticoagulant effects of edoxaban. Thromb Haemost 2017;117(02):238-245

112 Study of Ciraparantag Administered to Volunteers Anticoagulated With Rivaroxaban. Measure Clotting Times Using WBCT. ClinicalTrials.gov. Available at: https://clinicaltrials.gov/ct2/show/ NCT03172910. Updated May 30, 2017. Accessed July 6, 2017 